\title{
Pancreatic echinococcosis
}

\author{
Priti Soin, MBBS, MDª (D), Pranav Sharma, MBBS, MD ${ }^{b}$, and Puneet Singh Kochar, MBBS, MD ${ }^{b}$ (D) \\ ${ }^{a}$ Department of Pathology, GLM Hospital, Delhi, India; 'Department of Radiology, Yale New Haven Health Bridgeport Hospital, Bridgeport, \\ Connecticut
}

\section{ABSTRACT}

Echinococcosis (hydatid disease) is caused by the larval stage of Echinococcus granulosus. It most frequently involves the liver, followed by the lung. Pancreatic echinococcosis is very rare, even in endemic areas, with an incidence of $0.14 \%$ to $0.2 \%$. We describe a case of a 34-year-old woman who presented with epigastric pain for 1 month. Contrast-enhanced computed tomography demonstrated a $4.0-\mathrm{cm}$ hypodense mass in the pancreatic head and an uncinate process with suggestion of hyperdense/enhancing curvilinear densities. Magnetic resonance imaging with and without intravenous contrast showed a nonenhancing cystic mass. Endoscopic ultrasound demonstrated a cystic structure with curvilinear floating membranes consistent with the water lily sign, pathognomonic of hydatid disease. Enzyme-linked immunosorbent assay for echinococcal antigen was positive.

KEYWORDS Echinococcosis; hydatid disease; pancreas

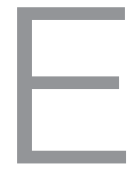

chinococcosis is a zoonotic disease caused by the larval stage of Echinococcus parasite. Four different species affect humans; the most common species are $E$. granulosus $(95 \%)$, which causes cystic echinococcosis, and E. multilocularis, which causes alveolar echinococcosis. Carnivores are the definitive hosts and herbivores are intermediate hosts in the life cycle of echinococcosis. Humans are inadvertently infected due to ingestion of vegetables contaminated with carnivores' feces. ${ }^{1}$ This disease is endemic to the Mediterranean region, Africa, South America, Australia, the Middle East, and India, where stockbreeding and agriculture are common occupations. ${ }^{2}$ Hydatid cysts can be seen in any organ, but the most common sites are the liver $(50 \%-77 \%)$ and lung $(15 \%-47 \%)$, followed by spleen $(0.5 \%-8 \%)$ and kidney (2\%-4\%). Pancreatic hydatids are very rare, even in endemic areas. ${ }^{3}$ In the pancreas, the most common location is the pancreatic head (57\%), followed by the body (24\%) and the tail (19\%). ${ }^{4}$

\section{CASE REPORT}

A 34-year-old woman presented with epigastric abdominal pain for 1 month radiating to the back. Her pain was insidious in onset, nonprogressive, continuous, and mild (3/10 intensity). Physical examination was noncontributory. There was no prior significant history. Her blood workup was normal, including serum amylase and lipase levels. Upper gastrointestinal endoscopy was normal. Ultrasound abdomen was advised, and the pancreas could not be assessed due to obscuration by overlying bowel gas (images not shown). Computed tomography (CT) with intravenous contrast demonstrated a $4.0 \times 3.5 \times 1.5 \mathrm{~cm}$ hypodense mass in the pancreatic head/ uncinate process. Within this mass, there was suggestion of hyperdense/enhancing curvilinear densities. It was uncertain whether these were dense/calcified septa or enhancement, because no precontrast imaging was performed (Figure 1). These findings were indeterminate, and a broad differential diagnosis was given that did not include hydatid disease.

Magnetic resonance imaging (MRI) of the abdomen with and without intravenous contrast demonstrated a $4.0-\mathrm{cm}$ mass appearing heterogeneously T2 hyperintense in the pancreatic head/uncinate process causing mild compression of the common bile duct. There was suggestion of linear T2 hypointensities within the cystic mass (Figures $2 a-2 c$ ). No enhancement was noted on contrast imaging (Figure 2d). Endoscopic ultrasound demonstrated a cystic structure with thin curvilinear floating membranes consistent with the "water lily sign," a classic pathognomonic sign of hydatid

Corresponding author: Puneet Singh Kochar, MBBS, MD, Department of Radiology, Yale New Haven Health Bridgeport Hospital, 267 Grant Street, Bridgeport, CT 06610 (e-mail: drkochar.puneet@gmail.com)

Color versions of one or more of the figures in this article can be found online at www.tandfonline.com/ubmc.

Received June 30, 2018; Revised August 6, 2018; Accepted August 13, 2018. 


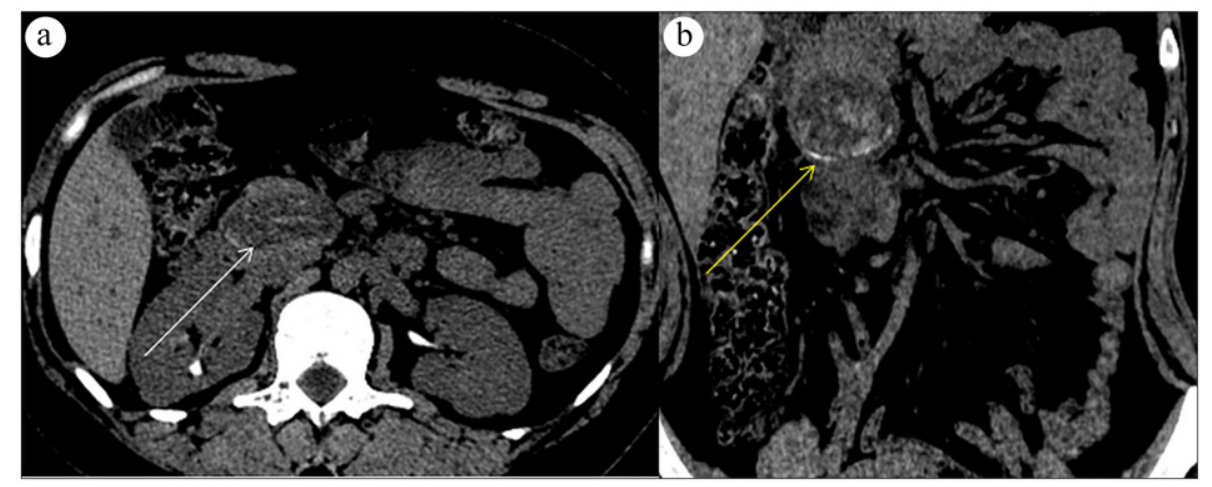

Figure 1. (a) CT scan with intravenous contrast demonstrates a 4.0-cm hypodense mass in the pancreatic head and uncinate process (white arrow). (b) There is the suggestion of hyperdense/enhancing curvilinear densities representing either hyperdense/calcified septa or enhancement (arrow), because no precontrast imaging was performed.

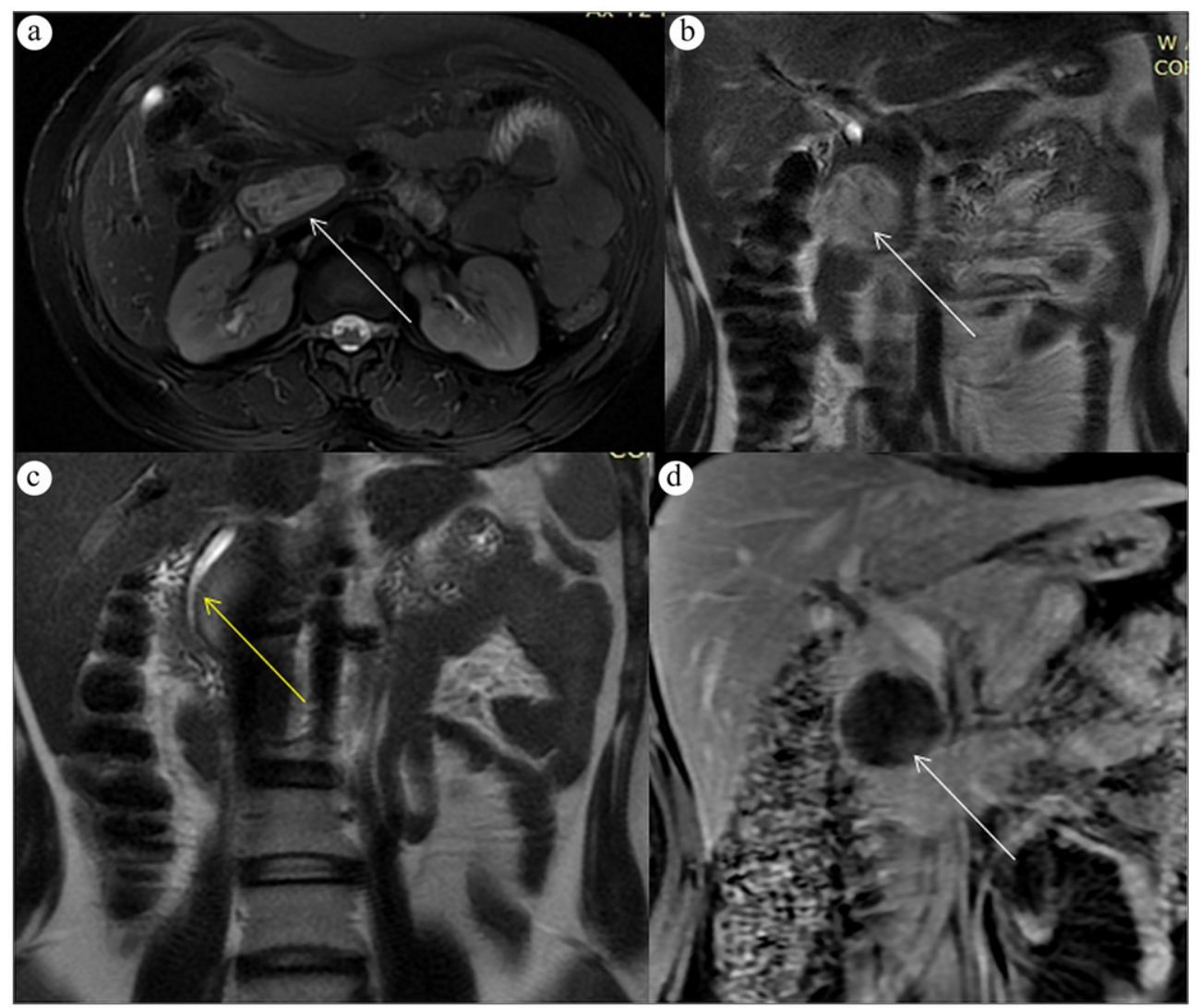

Figure 2. MRI of the abdomen with and without intravenous contrast shows $(\mathbf{a}, \mathbf{b})$ the mass appearing heterogeneously T2 hyperintense (T2-weighted images, white arrows), causing (c) splaying and mild attenuation of the common bile duct (T2-weighted image, arrow). (d) Postcontrast image demonstrates no enhancement (white arrow).

disease (echinococcosis) (Figure 3). Enzyme-linked immunosorbent assay (ELISA) for echinococcal antigen was positive. The patient was started on oral albendazole and was referred to surgery for further consultation and appropriate management.

Unfortunately, the patient was lost to follow-up, and attempts to contact her failed. Although a surgical specimen was not available to review, the presence of floating membranes within the matrix of the cyst and the positive ELISA test for echinococcal antigen confirmed the diagnosis.

\section{DISCUSSION}

Pancreatic echinococcosis is a very rare clinical entity, with an incidence ranging from $0.14 \%$ to $2 \% .^{5}$ In addition to the rarity of this condition, diagnosis is challenging due to the similarity of imaging with much more common pancreatic cystic masses. ${ }^{6}$ Most of these masses are solitary $(90 \%-91 \%){ }^{7}$

Hematogenous dissemination is thought to be the most common mode of spread to the pancreas. Other possible modes include passage through the biliary tract, lymphatic spread from the intestine, direct passage via the pancreatic veins, and dissemination along the retroperitoneum. ${ }^{1,7}$ 


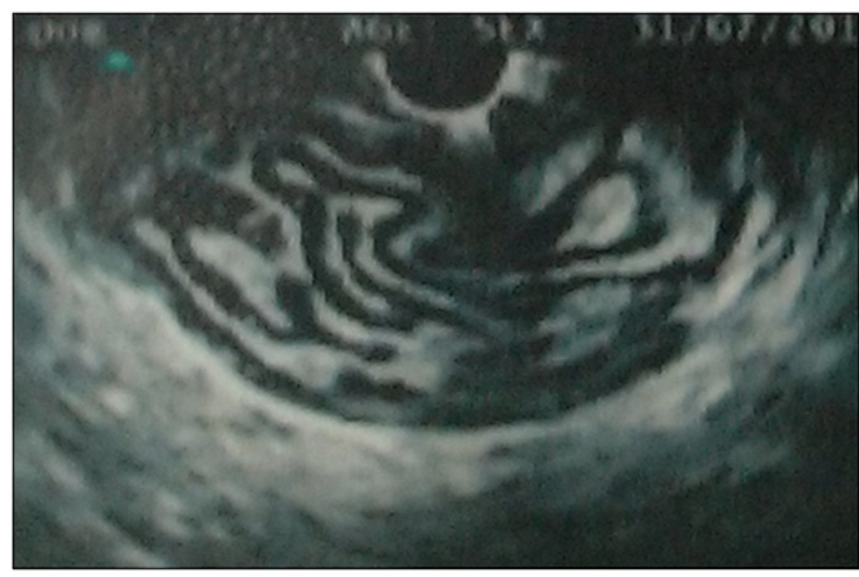

Figure 3. Endoscopic ultrasound image demonstrates a cyst with thin curvilinear hypoechoic floating membranes consistent with the water lily sign.

Pancreatic echinococcosis is usually asymptomatic, due to its slow growth rate of 0.3 to $2.0 \mathrm{~cm}$ per year. ${ }^{1}$ Clinical presentation depends on the location. Pancreatic head cysts usually present as obstructive jaundice due to extrinsic compression of the common bile duct and mimic a choledochal cyst. ${ }^{7,8}$ Pancreatic body and tail cysts stay asymptomatic and usually present as an abdominal lump when they enlarge. Additional symptoms include epigastric pain, nausea, and vomiting. ${ }^{1}$ Complications include cholangitis, rupture into the biliary system or peritoneal cavity, pancreatic fistula, recurrent pancreatitis, and pancreatic abscess formation.

Imaging plays an important role in diagnosis. Abdominal ultrasonography is a very sensitive imaging modality for diagnosing hydatid cysts. Characteristic sonographic findings include floating membranes and hydatid sand and daughter cysts; however, the sensitivity is low due to the deep retroperitoneal location and overlying bowel gas. ${ }^{7}$ Floating membranes and daughter cysts within a larger mother cyst can also be seen on CT and MRI to suggest diagnosis. ${ }^{5}$ Endoscopic ultrasound can better evaluate the nature of the pancreatic cyst. Floating membranes within the cyst as serpentine linear structures are highly specific for hydatid disease. ${ }^{9}$ Additionally, guided aspiration of cystic fluid can help to exclude pancreatic cystic neoplasms and pseudopancreatic cysts. ${ }^{2}$ Magnetic resonance cholangiopancreatography is helpful in evaluating the biliary tract and pancreatic duct when the cystic mass is located in the pancreatic head. ${ }^{10}$ Numerous blood tests are available for detecting specific serum antibodies and echinococcal antigens. ${ }^{1}$ The ELISA test for echinococcal antigen is usually positive, with a sensitivity of $93.5 \%$, specificity of $89.7 \%$, and diagnostic accuracy of $92.3 \% .{ }^{11}$ Newer ELISA techniques have sensitivity and specificity of $98.0 \%$ and $96.2 \%$, respectively. ${ }^{12}$

Surgery is the treatment of choice. ${ }^{1}$ Patients with a preoperative diagnosis of echinococcosis should always receive prophylactic anthelminthic agents (Albendazole $10 \mathrm{mg} / \mathrm{kg}$ / day) for 2 to 4 weeks, which should continue for at least 4 weeks after surgery to reduce the risk of anaphylaxis. ${ }^{1}$
Conservative management techniques like puncture-aspiration-injection-reaspiration or direct percutaneous catheterization with medical therapy have also been used for nonsurgical candidates. ${ }^{1,7}$

Pancreatic echinococcosis is an extremely rare clinical condition that can mimic more common cystic pancreatic lesions. Physicians in the Western world (nonendemic regions) who have not seen a pancreatic hydatid in their practice may not even consider it in the differential diagnosis. In nonendemic areas, a past history of traveling or immigration should make physicians suspect the possibility of hydatid disease. ${ }^{13}$

\section{ORCID}

Priti Soin (ID) http://orcid.org/0000-0002-6315-8281

Pranav Sharma (iD http://orcid.org/0000-0002-5141-7805

Puneet Singh Kochar (DD http://orcid.org/0000-0001-5213-0438

1. Akbulut S, Yavuz R, Sogutcu N, et al. Hydatid cyst of the pancreas: report of an undiagnosed case of pancreatic hydatid cyst and brief literature review. World J Gastrointest Surg. 2014;6(10):190-200. doi: 10.4240/wjgs.v6.i10.190.

2. Sharma S, Sarin H, Guleria M, Agrawal K. Endoscopic ultrasoundguided FNA: emerging technique to diagnose hydatid cyst of pancreas. J Cytol. 2015;32(3):211-212. doi:10.4103/0970-9371.168908.

3. Jai SR, El Hattabi K, Bensardi F, Chehab F, Khaiz D, Bouzidi A. Primary hydatid cyst of the pancreas causing obstructive jaundice. Saudi J Gastroenterol. 2007;13(4):191-193. doi:10.4103/1319-3767.36752.

4. Sorogy ME, El-Hemaly M, Aboelenen A. Pancreatic body hydatid cyst: a case report. Int J Surg Case Rep. 2015;6:68-70. doi:10.1016/ j.ijscr.2014.11.062.

5. Shah OJ, Robbani I, Zargar SA, et al. Hydatid cyst of the pancreas. An experience with six cases. JOP. 2010;11(6):575-581.

6. Dahniya $\mathrm{MH}$, Hanna RM, Ashebu $S$, et al. The imaging appearances of hydatid disease at some unusual sites. Br J Radiol. 2001;74(879): 283-289. doi:10.1259/bjr.74.879.740283.

7. Ahmed Z, Chhabra S, Massey A, et al. Primary hydatid cyst of pancreas: case report and review of literature. Int J Surg Case Rep. 2016; 27:74-77. doi:10.1016/j.ijscr.2016.07.054.

8. Turkyilmaz Z, Kapisiz A, Sonmez K, Karabulut R, Basaklar A. Obstructive jaundice resulted from hydatid cyst of pancreatic head. Hippokratia. 2013;17(4):378-379.

9. Von Sinner WN. New diagnostic signs in hydatid disease; radiography, ultrasound, CT and MRI correlated to pathology. Eur J Radiol. 1991;12(2):150-159.

10. Agrawal S, Parag P. Hydatid cyst of head of pancreas mimicking choledochal cyst. BMJ Case Rep. 2011;2011:bcr0420114087.

11. Sbihi Y, Rmiqui A, Rodriguez-Cabezas MN, Orduña A, RodriguezTorres A, Osuna A. Comparative sensitivity of six serological tests and diagnostic value of ELISA using purified antigen in hydatidosis. J Clin Lab Anal. 2001;15(1):14-18. doi:10.1002/1098-2825(2001)15: $1<14::$ AID-JCLA3 > 3.0.CO;2-7.

12. Sarink MJ, Koelewijn R, Slingerland BCGC, Tielens AGM, Van Genderen PJJ, Van Hellemond JJ. Performance of the commercially available SERION ELISA classic Echinococcus IgG test for detection of cystic echinococcosis in clinical practice. J Helminthol. 2018:1-4. doi:10.1017/S0022149X18000536.

13. Ozmen MM, Moran M, Karakahya M, Coskun F. Recurrent acute pancreatitis due to a hydatid cyst of the pancreatic head: a case report and review of the literature. JOP. 2005;6(4):354-358. 